\title{
Serum $\beta$-human chorionic gonadotropin and interleukin-1 as diagnostic biomarkers for the premature rupture of membranes and chorioamnionitis
}

\author{
CHUN-FANG TIAN ${ }^{1}$, FA-HUI LV ${ }^{2}$, MIN WANG ${ }^{1}$ and XIAO-SHAN GU ${ }^{1}$ \\ ${ }^{1}$ Department of Obstetrics and Gynecology, Shenzhen Seventh People's Hospital, Shenzhen, Guangdong 518081; \\ ${ }^{2}$ Department of Obstetrics and Gynecology, Hefei Second People's Hospital, Hefei, Anhui 230000, P.R. China
}

Received June 7, 2014; Accepted July 21, 2014

DOI: $10.3892 /$ br.2014.342

\begin{abstract}
Chorioamnionitis is common in females with prematurely ruptured fetal membranes (PROM). The current diagnosis of PROM and preterm PROM (PPROM) is based on vaginal fluid analysis. The present study investigated the value of serum $\beta$-human chorionic gonadotropin $(\beta$-hCG) and interleukin-1 (IL-1) levels in diagnosing chorioamnionitis. In total, 150 term-pregnancy patients were included in the prospective study. A total of 50 females had normal pregnancies (control group) and 100 had PROM. One hour before delivery, $3 \mathrm{ml}$ venous blood was collected and analyzed. Fetal membrane and placental tissue underwent histopathological analyses. Of the 100 term-pregnancy females, 56 had PROM and 44 had PROM combined with chorioamnionitis (PROM $+\mathrm{C})$. The serum $\beta$-hCG levels for the control, PROM and PROM + C groups were 7,557.86 $\pm 2,922.06,636.96 \pm 14,379.10$ and $50,310.34 \pm 22,874.82 \mathrm{IU} / 1$, respectively. The receiver operating characteristic (ROC) for PROM and PROM $+\mathrm{C}$ groups $(\beta-\mathrm{hCG}$ $\geq 23,900.50 \mathrm{IU} / \mathrm{l})$ had a sensitivity of $77.5 \%$ and a specificity of $78.6 \%$. The level of IL-1 in the PROM + C group was higher compared to the control and PROM groups $(0.58 \pm 0.05$, $0.12 \pm 0.04$ and $0.13 \pm 0.03 \mathrm{ng} / \mathrm{ml}$, respectively). In conclusion, ROC for the PROM and PROM + C groups (IL-1 $\geq 0.38 \mathrm{ng} / \mathrm{ml}$ ) had a sensitivity of $76.5 \%$ and a specificity of $72.6 \%$. Therefore, serum $\beta$-hCG and IL-1 are potential biomarkers for diagnosing $\mathrm{PROM}$ and PROM $+\mathrm{C}$, respectively.
\end{abstract}

\section{Introduction}

Premature rupture of membranes (PROM) and preterm PROM (PPROM) are common obstetric complications. PPROM is the

Correspondence to: Dr Chun-Fang Tian, Department of Obstetrics and Gynecology, Shenzhen Seventh People's Hospital, 2032 Wutong Street, Shenzhen, Guangdong 518081, P.R. China

E-mail: SZTCF888@163.com

Key words: premature rupture of membranes, chorioamnionitis, interleukin-1, serum $\beta$-human chorionic gonadotropin, $\mathrm{C}$-reactive protein rupture of membranes in a pregnant female prior to the onset of labor, before 37 weeks gestation and PROM occurs after 37 weeks gestation. The incidence of PPROM is $2.0-3.5 \%$ and the incidence of PROM is $10 \%$ (1). In particular, PPROM can cause neonatal sepsis, pneumonia and neonatal pulmonary hyaline membrane disease. All these secondary factors can increase the risk of perinatal fetal mortality.

The current diagnosis of PPROM and PROM is based on vaginal fluid analysis. However, samples are easily contaminated, resulting in a high rate of false positives. In addition, there is too little amniotic fluid in PROM to provide an adequate sample and this can result in a high false-negative rate. When PROM is combined with chorioamnionitis $(\mathrm{PROM}+\mathrm{C})$, the diagnoses made according to the $\mathrm{C}$-reactive protein (CRP) levels are not specific to intrauterine infections and may be influenced by other factors (1). Therefore, PPROM and PROM diagnostic procedures, particularly when combined with chorioamnionitis, are largely limited. The aim of the present study was to investigate the changes in serum $\beta$-human chorionic gonadotropin ( $\beta$-hCG) and interleukin-1 (IL-1) levels in PROM females and compare these with the levels in females with normal pregnancies. The results indicate that measuring serum $\beta$-hCG and IL-1 levels may provide an alternative diagnostic tool for determining infection in parturient females.

\section{Materials and methods}

Patient information. A total of 100 patients with PROM in the Department of Obstetrics and Gynecology at the Shenzhen Seventh Peoples Hospital (Shenzhen, Guangdong, China) were randomly selected for the prospective study. All the human participants provided written informed consent prior to the study. The study protocol was approved by the local Institutional Review Board or Ethics Committee prior to the study. A total of 56 patients had PROM only. The average gestational age for this group was $37-41^{+6}$ weeks, the patient age range was 20-35 years and the average age was $25 \pm 3.2$ years. The remaining 44 patients had PROM + C. Within this group, the average gestational age was $37-41^{+6}$ weeks, the patient age range was 20-35 years and the average age was $26 \pm 2.8$ years. Simultaneously, 50 females with normal pregnancies were chosen as the control group. The average gestational age was 
$37 \pm 41^{+6}$ weeks, the patient age range was 20-34 years and the average age was $24 \pm 3.4$ years. Females with any other obstetric complications were excluded from the study.

Blood analysis. A total of $3 \mathrm{ml}$ venous blood was drawn from all the patients $\sim 1 \mathrm{~h}$ before delivery. The blood samples were centrifuged for $15 \mathrm{~min}$ at $4,929 \mathrm{x}$ g to separate the serum. A chemiluminescence assay (Roche Diagnostics, Madison, WI, USA) was used to determine the $\beta$-hCG levels. The reagent was used according to the manufacturer's instructions (Randox Life Science, Crumlin, UK) and chemiluminescence was measured using a Cobas 6000 analyzer (Roche Diagnostics, Madison, WI, USA).

A turbidity test (Zhongguo, Shanghai, China) was used to provide a preliminary diagnosis of chorioamnionitis and to determine the serum CRP levels. The test was performed according to the manufacturer's instructions. ELISA (Shanghai Yisha Biological Technology Co., Ltd., Shanghai, China) was used to detect serum IL-1 levels. The results were measured using an auto-analyzer (Gold Innovative Materials Co., Ltd., Anhui, China).

Placenta and fetal membrane tissues. Following the delivery of the placenta, a membrane sample that was $5 \mathrm{~cm}$ from the membrane breach of each patient was obtained. Each sample size was $\sim 5 \times 5 \mathrm{~cm}$. Two placenta tissue samples that were $\sim 2 \times 2 \mathrm{~cm}$ in size were also obtained from each patient. One sample was from the placental edge and the other was collected from the center of the placenta. All the samples were fixed with $10 \%$ formalin prior to pathological analyses.

PROM and chorioamnionitis diagnoses. PROM was diagnosed using standard procedures (2). Briefly, the patient was diagnosed with PROM if any of the following clinical criteria were observed upon pelvic examination with a sterile speculum: Amniotic fluid leaving or pooling behind the cervix, the fluid had a $\mathrm{pH}>6.5$ and a vaginal posterior fornix smear exhibited a fern-like appearance under the microscope when dried.

The patients were diagnosed with chorioamnionitis using standard clinical and histopathological procedures (3). The clinical diagnoses were made according to malodorous amniotic fluid or vaginal discharge, maternal fever, uterine tenderness, increased white blood cell count and serum CRP levels $>8 \mathrm{mg} / \mathrm{l}$.

Histopathological diagnoses were generated and staged according to the following criteria: Stage I, minor neutrophil invasion without significant chorionic fibrin deposition of chorionic plate or limited to the decidual layer; stage II, significant neutrophil invasion extended to chorionic tissues and fetal blood vessels, but not the amniotic membrane; and stage III, extensive neutrophil invasion of the decidual, chorionic and amniotic membrane. Neutrophils covering the surface of the amniotic membrane indicated sepsis in the amniotic cavity. The patients were assigned a level based on neutrophil count: Level 1, $<10$ neutrophils; level II, 11-30 neutrophils; and level III, $>30$ neutrophils per field of view at high magnification (3). These observations indicated the severity of chorioamnionitis. The association between $\beta$-hCG levels and the severity of chorioamnionitis was not investigated in the study.
Statistical analysis. SPSS 17.0 software (SPSS, Inc., Chicago, IL, USA) was used to perform statistical analyses. Independent sample t-tests were used to analyze the patient characteristics. Receiver operating characteristic (ROC) curve construction was used for diagnostic test evaluation. Enumeration data was analyzed using the $\chi^{2}$ test. $\mathrm{P}<0.05$ was considered to indicate a statistically significant difference.

\section{Results}

Statistical analyses of patient parameters. Statistical analyses were performed for all the patient parameters between the control, PROM and PROM + C groups. There were no statistical differences between parity, gestational age and patient age for the three study groups $(\mathrm{P}>0.05)$. The patient characteristics are summarized in Table I.

To determine if there were any differences in serum $\beta$-hCG, CRP and IL-1 levels between the control and PROM groups, the blood samples that were obtained from all the patients $\sim 1 \mathrm{~h}$ before delivery were analyzed. The serum $\beta$-hCG levels were higher in the PROM compared to the control group $(18,636.96 \pm 14,379.10$ vs. $7,557.86 \pm 2,922.06 \mathrm{IU} / 1 ; \mathrm{P}<0.05)$. There were no significant differences in serum CRP and IL-1 levels between the two groups ( $\mathrm{P}>0.05$; Table II).

An ROC curve was constructed to test the diagnostic value of measuring serum $\beta$-hCG levels between patients in the control and PROM groups. The area under the ROC curve was 0.80 (Fig. 1). Therefore, serum $\beta$-hCG levels have a clinical value in diagnosing PROM. According to the ROC curve coordinates, when serum $\beta$-hCG levels were $\geq 8,382.50 \mathrm{IU} / 1$, the sensitivity of diagnosing PROM with this measurement was $71 \%$ and the specificity was $76 \%$.

Serum levels of $\beta$-hCG, CRP and IL- 1 . Whether there were any differences in serum $\beta$-hCG, CRP and IL-1 levels between the PROM and PROM $+\mathrm{C}$ groups was also investigated (Table III). The PROM $+\mathrm{C}$ serum $\beta$-hCG levels were higher compared to the PROM group $(50,310.34 \pm 22,874.82 \mathrm{IU} / 1$ and $18,636.96 \pm 14,379.10 \mathrm{IU} / 1$, respectively; $\mathrm{P}<0.05$ ). The area under the ROC curve constructed for the serum $\beta$-hCG levels in these two groups was 0.87 (Fig. 2). Therefore, the measurement of serum $\beta$-hCG levels has clinical value in distinguishing between PROM and PROM + C. According to the ROC coordinates, when serum $\beta$-hCG levels were $\geq 23,900.50 \mathrm{IU} / 1$, the sensitivity of diagnosing PROM $+\mathrm{C}$ with this measurement was $77.5 \%$ and the specificity was $78.6 \%$.

The differences in the serum CRP and IL-1 levels between the PROM and PROM $+\mathrm{C}$ groups were also statistically significant $(\mathrm{P}<0.05)$. The serum CRP levels for the PROM and PROM $+C$ groups were $6.76 \pm 1.75$ and $17.27 \pm 7.18 \mathrm{mg} / 1$, respectively. The serum IL-1 levels for the PROM and PROM + C groups were $0.13 \pm 0.03$ and $0.58 \pm 0.05 \mathrm{ng} / \mathrm{ml}$, respectively (Table III). According to ROC curve analyses, serum IL-1 levels can distinguish between patients with PROM alone and patients with PROM $+\mathrm{C}$. The ROC curve coordinates show that when serum IL-1 levels are $\geq 0.38 \mathrm{ng} / \mathrm{ml}$, the sensitivity of diagnosing PROM $+\mathrm{C}$ is $76.5 \%$ and the specificity is $72.6 \%$ (Fig. 3). This was compared to the sensitivity of diagnosing PROM $+\mathrm{C}$ according to serum CRP levels (81.3\%) and the specificity was $(70.2 \%)$. These diagnostic serum-CRP values 
Table I. Patient characteristics.

\begin{tabular}{|c|c|c|c|c|c|}
\hline \multirow[b]{2}{*}{ Group } & \multicolumn{3}{|c|}{ Parity, n } & \multirow{2}{*}{$\begin{array}{c}\text { Average } \\
\text { age, } \\
\text { years }\end{array}$} & \multirow{2}{*}{$\begin{array}{c}\text { Average } \\
\text { gestational age } \\
\text { weeks }\end{array}$} \\
\hline & 1 & 2 & 3 & & \\
\hline Control & 34 & 14 & 2 & $24 \pm 3.4$ & $39^{+2} \pm 1^{+1}$ \\
\hline PROM & 38 & 15 & 3 & $25 \pm 3.2$ & $38^{+6} \pm 1^{+4}$ \\
\hline $\mathrm{PROM}+\mathrm{C}$ & 32 & 11 & 1 & $26 \pm 2.8$ & $38^{+4} \pm 1^{+3}$ \\
\hline
\end{tabular}

PROM, prematurely ruptured fetal membranes; $\mathrm{C}$, chorioamnionitis.

Table II. Comparative blood analyses between the control and PROM groups.

\begin{tabular}{lccc}
\hline Group & $\beta$-hCG, IU/l & CRP, mg/l & IL-1, ng/ml \\
\hline Control & $7,557.86 \pm 2,922.06$ & $6.08 \pm 1.70$ & $0.12 \pm 0.04$ \\
PROM & $18,636.96 \pm 14,379.10$ & $6.76 \pm 1.75$ & $0.13 \pm 0.03$ \\
P-value & $<0.05$ & 0.65 & 0.54 \\
\hline
\end{tabular}

PROM, prematurely ruptured fetal membranes; $\beta$-hCG, $\beta$-human chorionic gonadotropin; CRP, C-reactive protein; IL-1, interleukin-1.

Table III. Comparative blood analyses between the PROM and $\mathrm{PROM}+\mathrm{C}$ groups .

\begin{tabular}{lccc}
\hline Group & $\beta$-hCG, IU/l & CRP, mg/l & IL-1, ng/ml \\
\hline PROM & $18,636.96 \pm 14,379.10$ & $6.76 \pm 1.75$ & $0.13 \pm 0.03$ \\
PROM + C & $50,310.34 \pm 22,874.82$ & $17.27 \pm 7.18$ & $0.58 \pm 0.05$ \\
P-value & $<0.05$ & $<0.05$ & $<0.05$ \\
\hline
\end{tabular}

PROM, prematurely ruptured fetal membranes; $\mathrm{C}$, chorioamnionitis; $\beta$-hCG, $\beta$-human chorionic gonadotropin; CRP, C-reactive protein; IL-1, interleukin-1.

of sensitivity and specificity were also compared to those of serum $\beta$-hCG values. CRP sensitivity was higher than that of $\beta$-hCG, however, the specificity was lower. Using the $\chi^{2}$ test, it was determined that the differences between these two indices had no statistical significance $(\mathrm{P}>0.05)$.

The study aimed to determine if a combination of two blood analysis indices could be used to improve the sensitivity and specificity of diagnosing patients with PROM $+C$. The clinical value of combining serum $\beta$-hCG and IL-1 level measurements to diagnose PROM $+\mathrm{C}$ was evaluated. The results show that combining these two indices had no statistical significance ( $\mathrm{P}>0.05$; Table IV).

\section{Discussion}

In recent years, much progress has been made in elucidating the multiple factors that contribute to PROM. These include structural changes in the amniotic membrane, infection and immunology (4). Among them, infectious factors are a primary

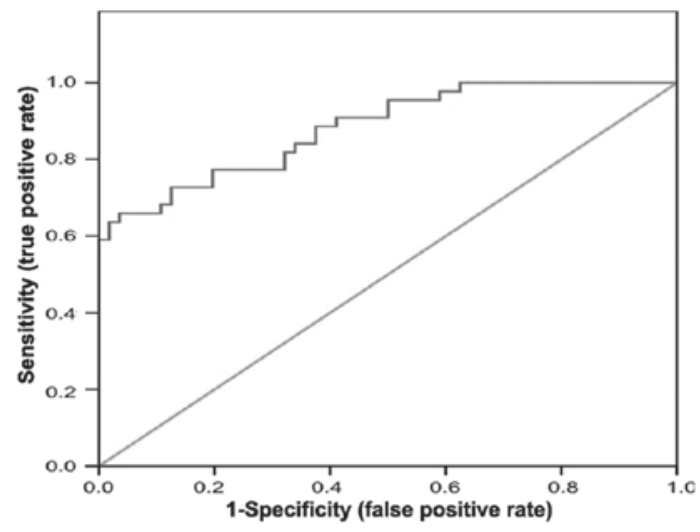

Figure 1. Receiver operating characteristic curve of serum $\beta$-human chorionic gonadotropin levels between the control and prematurely ruptured fetal membranes groups.

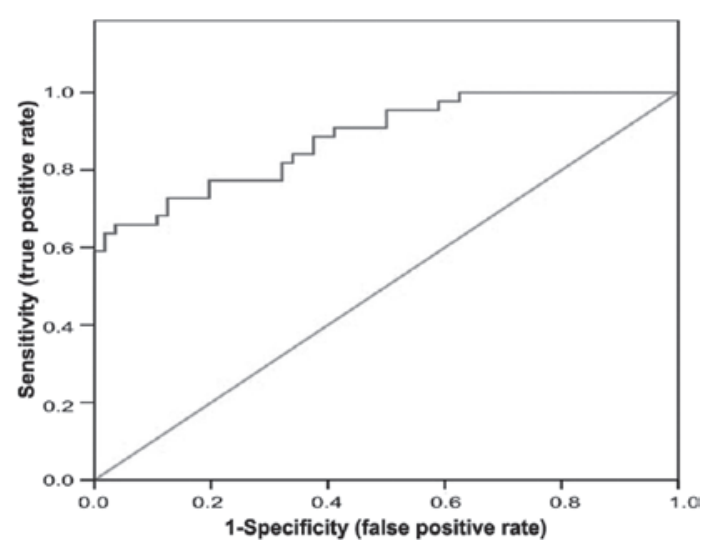

Figure 2. Receiver operating characteristic curve of serum $\beta$-human chorionic gonadotropin levels between the prematurely ruptured fetal membranes (PROM) and PROM with chorioamnionitis groups.

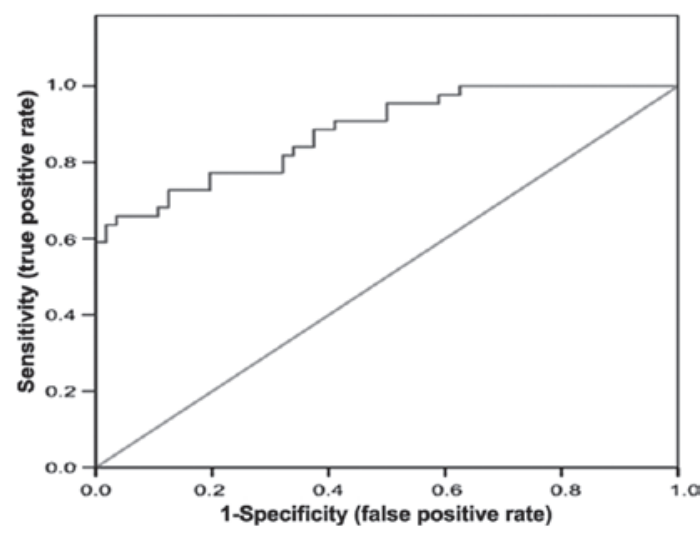

Figure 3. Receiver operating characteristic curve of serum interleukin-1 levels between the prematurely ruptured fetal membranes (PROM) and PROM with chorioamnionitis groups.

cause. Numerous pregnant females sustain infections prior and subsequent to amniotic membrane rupture and a number of these infections are asymptomatic. Therefore, it is important to diagnose PROM $+\mathrm{C}$ to improve maternal and fetal outcomes. The conventional diagnosis of PROM combines patient history, speculum examination, measuring vaginal fluid $\mathrm{pH}$ with 
Table IV. Evaluation of combined serum $\beta$-hCG and IL-1 ROC indices in the diagnosis of PROM $+\mathrm{C}$.

\begin{tabular}{lcccc}
\hline Index & Sensitivity, $\%$ & Specificity, $\%$ & Positive predictive value, $\%$ & Negative predictive value, $\%$ \\
\hline$\beta-\mathrm{hCG}, \geq 23,900.50 \mathrm{IU} / \mathrm{l}$ & 77.5 & 78.6 & 80.1 & 79.3 \\
$\mathrm{IL}-1, \geq 0.38 \mathrm{ng} / \mathrm{ml}$ & 76.5 & 72.6 & 78.3 & 77.6 \\
$\beta-\mathrm{hCG}+\mathrm{IL}-1$ & 77.8 & 79.7 & 81.1 & 78.6 \\
\hline
\end{tabular}

$\beta$-hCG, $\beta$-human chorionic gonadotropin; IL-1, interleukin-1; ROC, receiver operating characteristic; PROM, prematurely ruptured fetal membranes; $\mathrm{C}$, chorioamnionitis.

nitrazine paper and the cervical smear fern test (5). However, these tests are often subjective and have high false-positive rates due to vaginal contamination with blood, urine or semen (6). Non-invasive diagnostic tests include the detection of $\alpha$-fetoprotein, prolactin, insulin-like growth factor-binding protein-1 (IGFBP-1), placental $\alpha$ microglobulin-1 (PAMG-1) and $\beta$-hCG in vaginal fluids $(7,8)$. Bedside strip tests for IGFBP-1 and PAMG-1 are the most commonly used and most accurate assays, but a gold standard non-invasive diagnostic test for PROM does not yet exist (9).

In the current study, an alternative diagnostic test is reported for PROM in the detection of serum $\beta$-hCG levels. The levels were significantly higher in patients with PROM and PROM $+\mathrm{C}$, compared to females with normal pregnancies. When evaluated with an ROC curve, the data demonstrate that this application has clinical diagnostic value, as the sensitivity of diagnosing PROM is $71 \%$ and the specificity is $76 \%$.

The serum $\beta$-hCG levels rise quickly from conception until 8 -10 weeks gestation. At 4 months gestation, serum $\beta$-hCG levels progressively decline until delivery. As measuring serum $\beta$-hCG levels is relatively clear, this measurement alone or in combination with another diagnostic test, may be useful in diagnosing difficult cases of PROM. Conversely, the differences in serum CRP and IL-1 levels were not statistically significant in differentiating between patients with or without PROM. Therefore, these should not be considered as alternative diagnostic indices for PROM.

Early diagnosis of $\mathrm{PROM}+\mathrm{C}$ is pivotal in improving prognoses. Currently, chorioamnionitis diagnoses are performed by measuring CRP levels and by histopathology. Also, IL-6 is the most studied candidate and several studies have reached different conclusions. A study by Kopyra et al (10) indicated that detecting IL- 6 levels is more efficient than detecting CRP levels for diagnosing PROM $+\mathrm{C}$ and determining the severity of neonatal infection. However, Tita and Andrews (11) have shown that IL-6 plays a limited role in intrauterine infection and another study has shown that measuring serum IL-6 levels may be helpful in predicting subclinical chorioamnionitis (12).

The present study aimed to determine whether measuring serum $\beta$-hCG levels could be used as an alternative approach to differentiate between patients with PROM or PROM $+\mathrm{C}$. The data show that the serum $\beta$-hCG levels were significantly higher in patients with PROM $+\mathrm{C}$, when compared to patients that only had PROM. In addition, ROC curves indicated that this index has value as a clinical diagnostic tool (area under the curve $>0.5$ ). The sensitivity of diagnosing PROM $+\mathrm{C}$ with serum $\beta$-hCG levels was $77.5 \%$ and the specificity was $78.6 \%$. Although the data indicate that this diagnostic index is no more efficient than measuring CRP levels, the serum $\beta$-hCG levels are pregnancy-specific, whereas the CRP levels are not. In addition, Smith et al (13) have concluded that the detection of CRP levels alone cannot predict chorioamnionitis with certainty.

The study performed by Li (14) supports our hypothesis that serum $\beta$-hCG levels have a higher value in predicting chorioamnionitis compared to CRP levels. However, the pathophysiological mechanism in which serum $\beta$-hCG levels increase during infection has not yet been elucidated. When patients with PROM become infected, villous interstitial inflammatory cell infiltration and placental tissue injury occurs. This can lead to intrauterine hypoxia and cause reactive trophoblastic cell hyperplasia. This may result in increased synthesis and release of $\beta$-hCG (14). Another possibility is that intrauterine infection induces inflammatory cells to release more cytokines, such as IL-6 (15). In turn, circulating cytokines may induce trophoblast cells to produce more $\beta$-hCG. The severity of chorioamnionitis, as determined by histopathological grading, may be associated with increased $\beta$-hCG levels.

IL-1 is a cytokine that is primarily produced by monocytes. Using radiation immunology technology, our previous study found that the IL-1 receptor is highly expressed in the nerve fibers of the rat hippocampus, brain cortex, bulbus olfactorius, cerebellum choroid plexus, hypothalamus, corpus striatum and medulla oblongata (1). IL-1 is produced earlier than other host defense proteins and has a higher sensitivity and specificity. In the present study, serum IL-1 levels were significantly higher in patients with PROM $+\mathrm{C}$ when compared to patients with PROM only. Notably, a previous study demonstrated that the IL-1 levels in amniotic fluid are higher during pregnancy and IL-1 levels may be linked to an increased risk of PROM (16). In addition, a study by Puchner et al (17) demonstrated that the IL-1 $\beta$ levels in amniotic fluid are positively associated with preterm delivery. For every unit increase in IL-1 $\beta$, females are 7.2 times more likely to deliver preterm.

The present study demonstrates that the serum $\beta$-hCG and IL-1 levels are potential biomarkers for diagnosing PROM $+C$. The serum $\beta$-hCG levels may also be able to diagnose patients that only have PROM. $\beta$-hCG is a pregnancy-specific hormone, therefore it has an advantage in diagnosing PROM and $\mathrm{PROM}+\mathrm{C}$. In addition, blood samples are easy to obtain and analyses are cost effective. Using $\beta$-hCG, it may be possible to dynamically monitor PROM and detect chorioamnionitis 
at an early stage. The IL-1 response is rapid and inflammation-specific, adding to its value as a clinical diagnostic tool. Measuring the IL-1 levels is also cost effective. However, the use of these indices for diagnosing PROM $+\mathrm{C}$ resulted in a sensitivity and specificity $<80 \%$. Sensitivity and specificity must be improved prior to combining serum $\beta$-hCG and IL-1 levels for use as an effective diagnostic tool. However, serum $\beta$-hCG and IL-1 may be powerful diagnostic tools for $\mathrm{PROM}+\mathrm{C}$ when evaluated in parallel with other factors. Therefore, further studies are required to investigate this possibility.

\section{References}

1. Le J (ed): Obstetrics and Gynecology. 7th edition. Beijing People's Medical Publishing House, Beijing, pp137-138, 2008.

2. Falagas ME, Avgeri SG, Matthaiou DK, Dimopoulos G and Siempos II: Short- versus long-duration antimicrobial treatment for exacerbations of chronic bronchitis: a meta-analysis. J Antimicrob Chemother 62: 442-450, 2008.

3. Chen ZN (ed): Pathology of Obstetrics and Gynecology. Shanghai Medical University Press, Shanghai, pp343-344, 1996.

4. Malak TM, Ockleford CD, Bell SC, et al: Confocal immunofluorescence localization of collagen types I, III, IV, VI and their ulrtastructural organization in term human fetal membranes. Placenta 14: 385-406, 1993.

5. Casey ML and MacDonald PC: Interstitial collagen synthesis and processing in human amnion: a property of the mesenchynal cells. Biol Reprod 55: 1253-1260, 1996.

6. Menon R and Fortunato SJ: The role of matrix degrading enzymes and apoptosis in rupture of membranes. J Soc Gynecol Investig 11: 427-437, 2004.
7. Carranza Lira S, Negrete López M, Quinzaños Fresnedo C and Leaños Miranda A: Usefulness of the qualitative detection of hCG in cervicovaginal lavage for the diagnosis of premature rupture of membranes. Ginecol Obstet Mex 77: 142-146, 2009 (In Spanish).

8. Knapik D and Olejek A: Analysis of cervicovaginal fluid in the diagnosis of premature rupture of membranes. Ginekol Pol 82: 50-55, 2011 (In Polish).

9. van der Ham DP, van Teeffelen AS and Mol BW: Prelabour rupture of membranes: overview of diagnostic methods. Curr Opin Obstet Gynecol 24: 408-412, 2012.

10. Kopyra P, Seremak-Mrozikiewicz A and Drews K: Usefulness of PCT, IL-6, CRP measurement in the prediction of intraamniotic infection and newborn status in pregnant women with premature rupture of membranes. Ginekol Pol 81: 336-341, 2010 (In Polish).

11. Tita AT and Andrews WW: Diagnosis and management of clinical chorioamnionitis. Clin Perinatol 37: 339-354, 2010.

12. Hou LT, Zhou YW and Liu CF: Monitoring the significance of serum CRP and IL-1 in preterm premature rupture of membranes pregnant women. Chin J Mater Child Heal Care 5: 1777-1778, 2008.

13. Smith EJ, Muller CL, Sartorius JA, White DR and Maslow AS: C-reactive protein as a predictor of chorioamnionitis. J Am Osteopath Assoc 112: 660-664, 2012.

14. Li HX: Comparing serum $\beta$-hCG and CRP levels in predicting intrauterine infection and prognosis. Chin J Hosp Infect 22: 2016-2018, 2012.

15. Yu L and Chen JH: The relationship among CRP, preterm premature rupture of membranes and subclinical chorionic amnionitis. J Pract Med Tech 15: 2889-2890, 2008.

16 . Bitner A and Kalinka J: IL- $\beta$, IL- 6 promoter, TNF- $\alpha$ promoter and IL-1RA gene polymorphisms and the risk of preterm delivery due to preterm premature rupture of membranes in a population of Polish women. Arch Med Sci 6: 552-557, 2010.

17. Puchner K, Iavazzo C, Gourgiotis D, et al: Mid-trimester amniotic fluid interleukins (IL-1 $\beta$, IL-10 and IL-18) as possible predictors of preterm delivery. In Vivo 25: 141-148, 2011. 